\title{
AN EFFICIENT CLASSIFICATION \& SELECTION OF HUMAN ACTION USING TSVM
}

\author{
Asmita Bhaumik', Arun Biradar ${ }^{2}$, Sulaj Saha ${ }^{3}$ \\ ${ }^{1}$ Student, Computer science \& Engineering, East west institute of technology, Karnataka, India \\ ${ }^{2} \mathrm{HOD}$, Computer Science \& Engineering, East West Institute Of Technology, Karnataka, India \\ ${ }^{3}$ Intern Student, Electronics \& communication Engineering, Maven Silicon, Karnataka, India
}

\begin{abstract}
Presently a days human PC collaboration has turn into a major scope of uses for substance in feature examination, observation of action developments, and human cooperation with PC. In the Existing work human activities perceived by an arrangement of activity units which is called moderate ideas that can be considered from the preparation information. This work has exhibited a low name provincially weighted word setting descriptor for enhancing the interest-point-based representation which was utilized generally. Here, the proposed framework utilizes a descriptor that can fuse the data which are neighbour finely. In proposed work the GNMF-based activity units are utilizing to extension the semantic hole in representation of activity. Besides here another joint 12,1 -standard based inadequate model for activity unit choice in a style by separation where SVM is embraced as the premonition model for segregation word reference study and arrangement. It is the hyper plane based which can augment the detachment edge between two classes by utilizing the named specimens which are accessible. In any case, in some genuine applications, we are achieving marked examples which are excessive, while extensive estimated unlabeled specimens are effortlessly accessible. This is the reason we propose a Transductive Support vector machine, TSVMs are predominantly a calculations which is persistent and continually look the hyper plane of detachment in the element space with a transductive process by joining unlabeled examples in the preparation stage. The Scale Invariant Feature Transform (SIFT) is a calculation which is utilized to identify and portray scale-, interpretation and turn invariant neighbourhood highlights in pictures. The first SIFT calculation has been effectively connected by and large question recognition and acknowledgment assignments. One of its later uses additionally incorporates face acknowledgment, where it was demonstrated to convey empowering results. Filter based face acknowledgment strategies found in the writing depend vigorously on the supposed key point identifier, which finds interest focuses in the given picture that are eventually used to register the SIFT descriptors.
\end{abstract}

Keywords: SVM, TSVM Classifier, SIFT Algorithm, Bad points, Action Units.

\section{INTRODUCTION}

Vision-based human activity acknowledgment is the procedure of naming picture successions with activity marks. Hearty answers for this issue have applications in spaces, for example, visual observation, feature recovery and human-computer association. The assignment is trying because of varieties in movement execution, recording settings and between individual contrasts. In this study, we expressly address these difficulties. We give an itemized diagram of current advances in the field. Picture representations and the consequent grouping procedure are examined independently to concentrate on the oddities of late research. Besides, we talk about impediments of the cutting edge and framework promising bearings of examination. It intends to perceive the activities and objectives of one or more specialists from a progression of perceptions on the operators' activities and the ecological conditions. Since the 1980s, this exploration field has caught the consideration of a few software engineering groups because of its quality in giving customized backing to various applications and its association with a wide range of fields of study, for example, pharmaceutical, human-PC cooperation, or humanism.
To comprehend movement acknowledgment better, consider the accompanying situation. An elderly man awakens day break in his little studio flat, where he stays alone. He lights the stove to make a pot of tea, switches on the toaster broiler, and takes some bread and jam from the cabinet. After he takes his morning drug, a PC created voice tenderly reminds him to kill the toaster. Soon thereafter, his girl gets to a safe site where she filters a registration, which was made by a sensor arrange in her dad's loft. She observes that her dad is eating typically, taking his pharmaceutical on calendar, and keeping on dealing with his day by day life all alone. That data comforts her psyche.

A wide range of uses have been mulled over via specialists in movement acknowledgment; samples incorporate helping the debilitated and handicapped. For instance, Pollack et al.[1] demonstrate that via naturally observing human exercises, home-based restoration can be accommodated individuals experiencing traumatic mind wounds. One can discover applications running from security-related applications and logistics backing to area based administrations. Because of its numerous faceted nature, diverse fields may allude. 


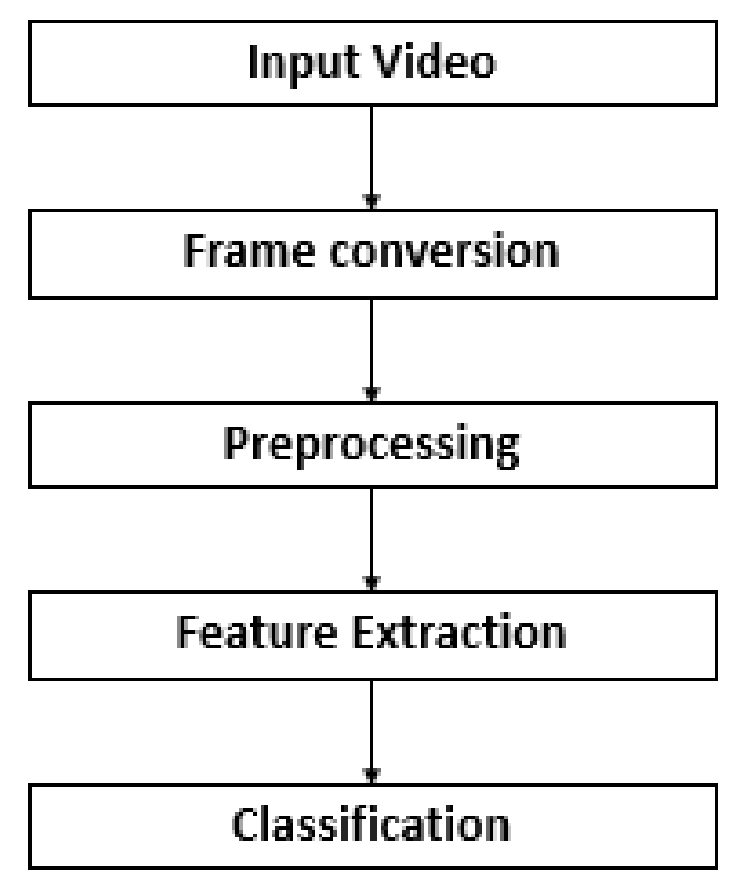

Fig 1: Architectural Flow chart of working process.

\section{COMPARISON}

SVM cannot learn the information about test set. Support vector machine can classify the labelled sample only, its not suitable for unlabeled sample TSVMs improved prediction accuracy as compared to the standard SVMs. Only support vector-like samples that contain the richest information are included in the training set. So the proposed system is well in classification result. Proposed technique is effective for the classification

\section{RELATED WORK}

\section{Human Action Categorization in Unsupervised}

\section{Method}

The undertaking of programmed arrangement and restriction of human activities in feature groupings is exceedingly fascinating for a mixture of utilizations: distinguishing significant exercises in reconnaissance feature, compressing and indexing feature arrangements, sorting out a computerized feature library as indicated by pertinent activities, and so forth. It remains, be that as it may, a testing issue for PCs to accomplish strong activity acknowledgment because of jumbled foundation, camera movement, impediment, perspective point changes, and geometric and photometric differences of articles. This work proposes an unsupervised learning methodology for human activities utilizing a pack of words representation. This work apply two inactive subject models, pLSA and LDA, to the issue of learning and perceiving human activity classifications, while embracing a "sack of spatial-fleeting words" representation for feature arrangements. Second, this technique can restrict and classify different activities in a solitary feature. Notwithstanding the order assignment, this methodology can likewise confine distinctive activities at the same time in a novel and complex feature grouping. This incorporates the situations where different individuals are performing particular activities in the meantime, furthermore circumstances where a solitary individual is performing unmistakable activities through time. Keeping in mind the end goal to accumulate exploratory confirmation that backings this proposed methodology, This work prepare and perceive activity models on three diverse datasets.

\section{Favorable Circumstances}

The proposed calculation can perceive and limit numerous activities in long and complex feature successions containing different movements.Generative strategy gives better knowledge into the comprehension of the activity classifications.

\section{Inconveniences}

Need to examine the conceivable outcomes of utilizing a bound together system by consolidating generative and discriminative models for human activity acknowledgment

\section{Action Recognition from Video}

This work shows a precise structure for re-cognizing reasonable activities from features "in nature." Such unconstrained features are bounteous in individual accumulations and on the web. Perceiving activity from such features has not been tended to broadly, principally because of the huge varieties that outcome from camera movement, foundation disarray, changes in article appearance, and scale, and so on. The fundamental test is the way to concentrate solid and instructive components from the unconstrained features. This work removes both movement and static elements from the features. Since the crude components of both sorts are thick yet uproarious, This work propose systems to prune these elements. This work utilization movement insights to secure stable movement elements and clean static components. Besides, Page Rank is utilized to mine the most educational static components. Keeping in mind the end goal to further build reduced yet discriminative visual vocabularies, a divisive data theoretic calculation is utilized to gathering semantically related components. At long last, AdaBoost is decided to coordinate all the heterogeneous yet reciprocal components for acknowledgment.

\section{Preferences}

It accomplishes better acknowledgment result. PR can adequately find the useful elements.

The proposed structure is viable for perceiving the practical activities.

\section{Detriments}

The significant trouble in distinguishing hearty movement components caught by a moving or shaking camera is jumpy movement, which may last just for a couple o 


\section{Activity Classification Based on Motion}

\section{Segmentation}

This work propose a straightforward discriminative structure for ordering human exercises by amassing data from movement sections that are viewed as both for their visual components and their worldly arrangement. A data feature is consequently disintegrated transiently into movement portions of variable lengths. The classifier chooses a discriminative deterioration and blend of the portions for coordinating. Despite the fact that straightforward in its frame, This work highlight a few favorable circumstances of this structure contrasted with the past work. Initially, contingent upon the time size of the development, activities have been generally gathered into: short however prompt activities (e.g. beverage, embrace), straightforward yet occasional activities (e.g. strolling, boxing), and more perplexing exercises that are considered as a creation of shorter or easier activities (e.g. a long hop, cooking). Altogether different calculations have been proposed for these distinctive sorts of movement, a large portion of them exploit the extraordinary properties inside of its dofundamental, consequently perform somewhat inadequately on different sorts. This system is a general one. Regardless of how basic or complex the movement is, this classifier depends on a transient creation of different movement fragments. This fundamental logic is clear: worldly data helps activity acknowledgment at unequaled scales.

\section{Points of Interest}

The execution of the proposed system is superior to different strategies

Selecting the theory with the best coordinating score should be possible anciently utilizing element programming and separation change procedures

\section{Detriments}

Classification precision is marginally low

Need to fuse different sorts of relevant data and wealthier feature represent.

\section{Various Feature Based Action Recognition}

This work proposes a structure those breakers different elements for enhanced activity acknowledgment in features. The combination of various components is essential for perceiving activities as frequently a solitary component based representation is insufficient to catch the imaging varieties (perspective point, brightening and so forth.) and characteristics of people (size, age, sex and so on.). Henceforth, This work utilizes two sorts of components: i) a quantized vocabulary of neighborhood spatio-worldly (ST) volumes (or cuboids), and ii) a quantized vocabulary of twist pictures, which intends to catch the shape distortion of the performing artist by considering activities as 3D articles ( $\mathrm{x}$, $y, t)$. To ideally consolidate these elements, This work regard distinctive components as hubs in a diagram, where weighted edges between the hubs speak to the quality of the relationship between substances. This work exhibits that by inserting these components into a typical Euclidian space, this work can find connections among them and can utilize them to enhance activity acknowledgment. The structure is general in nature and can be utilized with any kind of components.

\section{Points of Interest}

This methodology meets expectations extensively better for the activities that include full body movement, similar to in "walk", "get" and "pivot", while gets confounded in real life including just a piece of the body, similar to in "point", "cross arms" and "scratch head".

It accomplishes normal precision

\section{Burdens}

The variety of measurement $\mathrm{k}$ of the implanting space influences the exec.

\section{Spatiotemporal Feature Point on Saliency}

A few spatiotemporal component point identifiers have been as of late utilized as a part of feature examination for activity acknowledgment. Highlight focuses are identified utilizing various measures, in particular saliency, cornerness, periodicity, movement action and so on. Each of these measures is typically force based and gives an alternate exchange off in the middle of thickness and informativeness. This work introduced a novel spatiotemporal component point identifier, which is in light of a computational model of saliency. Saliency is gotten as the arrangement of a vitality minimization issue that is started by a situated of volumetric element conspicuities got from power, shading and movement. The vitality is compelled by terms identified with spatiotemporal vicinity, scale and comparability and highlight focuses are recognized at the extrema of the saliency reaction. Foundation commotion is naturally smothered because of the worldwide advancement system and in this way the recognized focuses are sufficiently thick to speak to well the fundamental activities. This work exhibits these properties in real life acknowledgment utilizing two assorted datasets.

\section{Favorable Circumstances}

It accomplishes better execution

Detector accomplishes the best results among routines depending on NNC and is second best among all.

\section{Detriments}

As expected, the worldwide precision of all finders is low Because of the differences of the substance. The quantity of codeword's expected to accomplish the higher rate shows the discriminative force of each recognized component. 


\section{WORKING MODULES}

\subsection{Input}

In this procedure, the info is the feature which is taken from the dataset. The info feature is otherwise called the crude data. It may be comprise of some clamor and loss of pixels.

The info feature may be in the organization of ".avi" or in the arrangement of.

\subsection{Preprocessing}

- $\quad$ Preprocessing is the procedure of changing over the single feature data to the $n$ number of casings.

- Frames is only the change of feature into the quantity of picture successions.

- On the other hand, it is the procedure of change of huge number of feature lattice into the quantity of little picture network.

- As well as pre-processing comprise of commotion separating.

- In the commotion separating process, the clamor is expelled from the changed oven

\subsection{Feature Extraction (SHIFT Algorithm)}

In feature extraction process the input video is converted into numeric values. The full video they will take as the input ant and later it will convert into numeric or may be decimal values.

\section{Constructing a scale space}

Scale space is the main starting point of SIFT algorithm. Here, pixel values are converted into Gaussian operator using scale space. The picture is blurred into various octaves formulation than it is converted into scale space format. The construction is done into format they need to use the blurring calculation of image.

$$
\mathrm{L}(\mathrm{x}, \mathrm{y}, \sigma)=\mathrm{G}(\mathrm{x}, \mathrm{y}, \sigma)^{*} \mathrm{I}(\mathrm{x} . \mathrm{y})
$$

The symbols:

- $\quad \mathrm{L}=$ Blurred Image

- $\mathrm{G}=$ Gaussian Blur operator

- $\quad \mathrm{I}=$ Image

- $\quad \mathrm{x}, \mathrm{y}=$ location coordinates

- $\sigma=$ "scale" parameter. Think of it as the amount of blur. Greater the value, greater the blur.

- $\quad$ The $*=$ convolution operation in $\mathrm{x}$ and $\mathrm{y}$. It "applies" Gaussian blur $\mathrm{G}$ onto the image I.

\section{LoG Approximation}

The Log Approximation is used for finding the main point in the image, that we can calculate the actual view recognition. The Log approximation is very much important in this feature because the only way to get the main analysis is the $\log$ approximation.
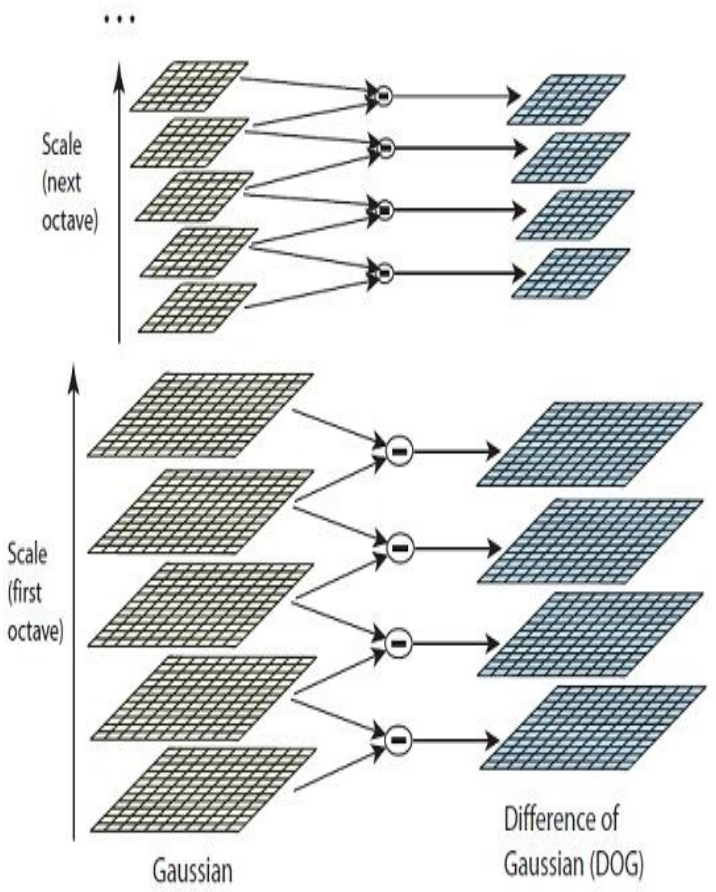

Fig 2: scale generation

These are the scales used for finding the interest points. It is calculating from the different label scales used in the feature extraction. So the different scale features are calculated.

\section{Detection of blurr point}

difference of blurr point
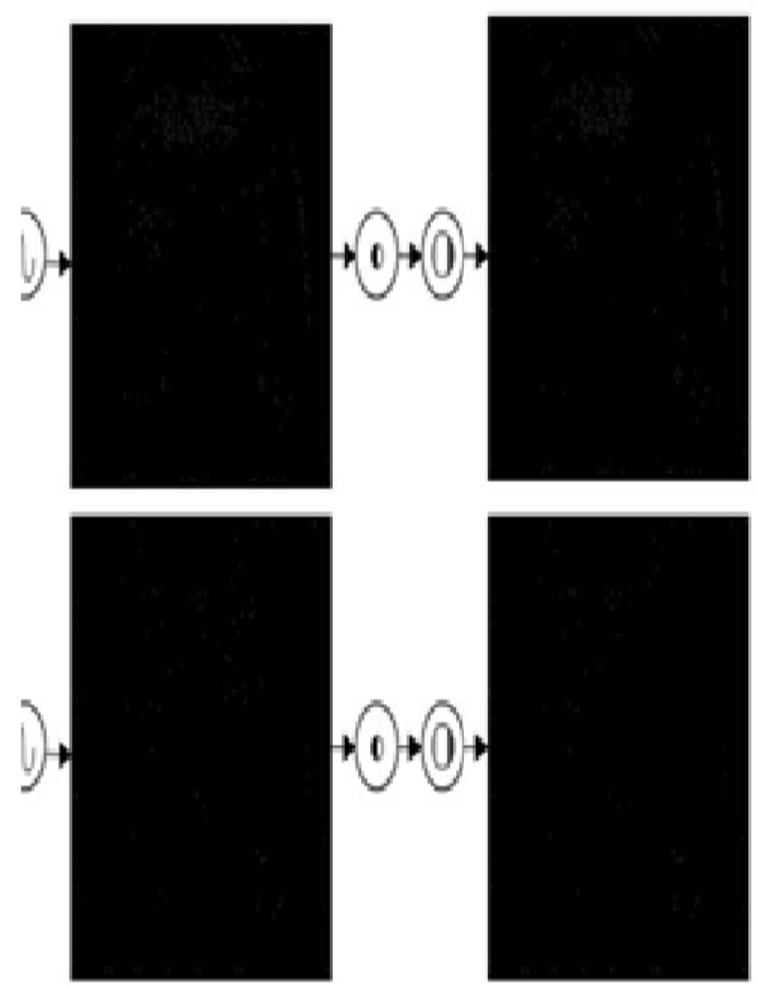

Fig 3: bad point reduction 


\section{Assigning an Orientation to the Key Points}

It is the orientation of key points. The key points are generated into format that the key is generated into pixel variations. The pixel variation is done here in the orientation.

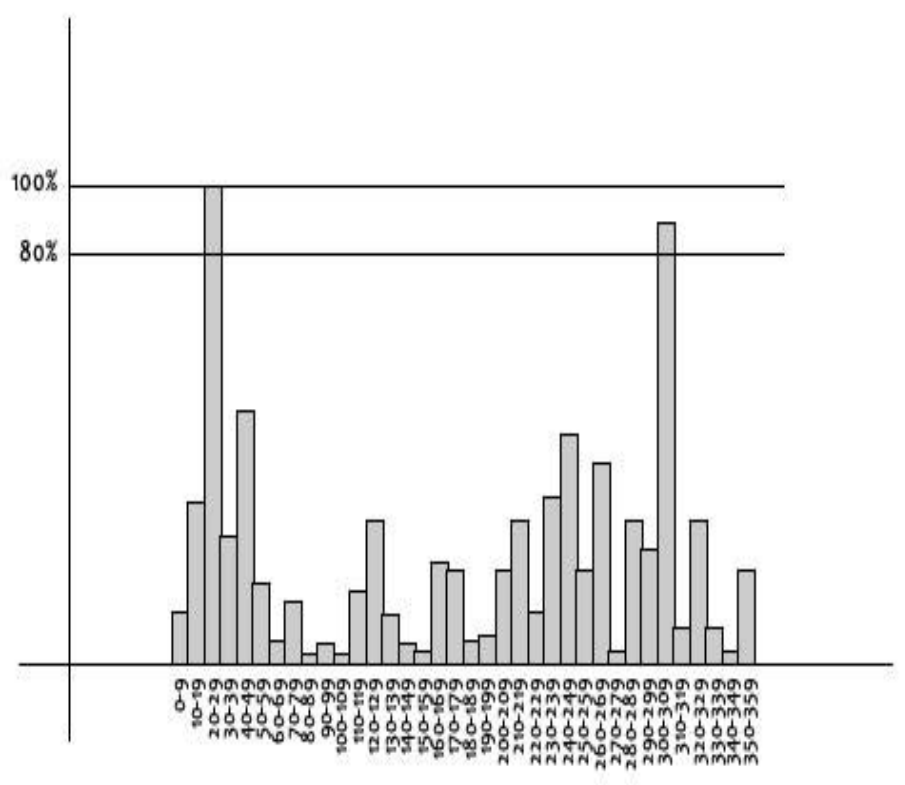

Fig 4: Gaussian blur image analysis.

\section{Generate SIFT Features}

This is the final presentation of SIFT algorithm. The pixels are converted into vector format here, the vector presentation is done at the end of this algorithm.

Now lastly the use of SIFT algorithm is come as histogram format. The histogram is generated.
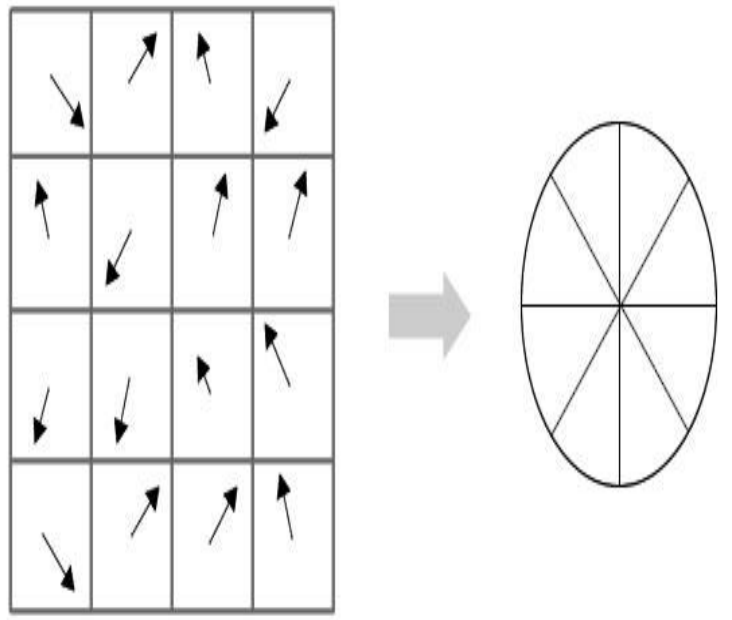

\subsection{Classification}

It is a process of classifying the test feature with the train feature. Here in classification we are using TSVM classifier where test feature is matching with the train feature.

\subsection{Performance Analysis}

In performance analysis the total system performance is calculated. The best comparison between the existing system and proposed system are shown here.

\section{CONCLUSION}

Transductive system can be used as an additional source margin compare to inductive system. This work can handle lots of examples on same test collection. This approach can show improvements on all other systems. Mostly the small examples can be done. Transactive system is much better than other system in all the sources.

\section{REFERENCES}

[1]. P. Turaga, R. Chellappa, V. S. Subrahmanian, and O. Udrea, "Machine recognition of human activities: A survey," IEEE Trans. Circuits Syst. Video Technol., vol. 18, no. 11, pp. 1473-1488, Sep. 2008.

[2]. J. C. Niebles, H.Wang, and L. Fei-Fei, "Unsupervised learning of human action categories using spatial-temporal words," in Proc. Int. J. Comput. Vis., vol. 79, no. 3, pp. 299318, Sep. 2008.

[3]. J. Liu, J. Luo, and M. Shah, "Recognizing realistic actions from videos 'in the wild'," in Proc. IEEE Int. Conf. Comput. Vis. Pattern Recognit., Jun. 2009, pp. 1996-2003.

[4]. J. Niebles, C. Chen, and L. Fei-Fei, "Modeling temporal structure of decomposable motion segments for activity classification," in Proc. Eur. Conf. Comput. Vis., 2010, pp. 392-405.

[5]. H. Wang, C. Yuan, W. Hu, and C. Sun, "Supervised class-specific dictionary learning for sparse modeling in action recognition," Pattern Recognit., vol. 45, no. 11, pp. 3902-3911, 2012.

[6]. J. Liu, B. Kuipers, and S. Savarese, "Recognizing human actions by attributes," in Proc. IEEE Int. Conf. Comput. Vis. Pattern Recognit., Jun. 2011, pp. 3337-3344.

[7]. J. Liu, S. Ali, and M. Shah, "Recognizing human actions using multiple features," in Proc. IEEE Int. Conf. Comput. Vis. Pattern Recognit.,

Fig 5: Histogram Generation 